\title{
Nile Red derivatives enable improved ratiometric imaging for nerve-specific contrast
}

Jesse R. Korber

Connor W. Barth

Summer L. Gibbs 


\title{
Nile Red derivatives enable improved ratiometric imaging for nerve-specific contrast
}

\author{
Jesse R. Korber, ${ }^{a}$ Connor W. Barth, ${ }^{a}$ and Summer L. Gibbs ${ }^{a, b, c, *}$ \\ ${ }^{\mathrm{a} O r e g o n ~ H e a l t h}$ and Science University, Department of Biomedical Engineering, Portland, Oregon, United States \\ bOregon Health and Science University, Knight Cancer Institute, Portland, Oregon, United States \\ 'Oregon Health and Science University, OHSU Center for Spatial Systems Biomedicine, Portland, Oregon, United States
}

\begin{abstract}
Surgical nerve damage due to difficulty with identification remains a major risk for postsurgical complications and decreased quality of life. Fluorescence-guided surgery offers a means to specifically highlight tissues of interest such as nerves and a number of fluorescence-guided surgical systems are in clinical trial or are approved for clinical use. However, no clinically approved nerve-specific fluorophores exist. In addition, many preclinical nerve-specific fluorophores tend to accumulate in adipose tissue due to the molecular composition similarities between the two tissues, making it challenging to generate a specific nerve signal. To alleviate this difficulty, we have synthesized a library of oxazine fluorophores based on the Nile Red scaffold, with the goal of strong adipose specificity without nerve uptake to facilitate ratiometric imaging. The library was screened for tissue specificity ex vivo and in vivo, enabling quantification of adipose-, nerve- and muscle-specific uptake as well as selection of the best candidate for adipose selectivity without nerve signal. We showed our selected Nile Red fluorophore improved nerve contrast using ratiometric imaging, especially nerve-to-adipose contrast as compared to the parent Nile Red compound or nerve-specific imaging alone. This adipose-specific Nile Red derivative could be used in future fluorescence-guided surgery applications where adipose- or nerve-specific contrast is required. ๑ 2018 Society of Photo-Optical Instrumentation Engineers (SPIE) [DOI: 10.1117/1.JBO.23.7.076002]
\end{abstract}

Keywords: fluorescence-guided surgery; nerve-specific fluorophores; Nile Red derivatives.

Paper 180172R received Mar. 21, 2018; accepted for publication Jun. 11, 2018; published online Jul. 6, 2018.

\section{Introduction}

Nerve damage sustained during surgery remains a major risk to patients, resulting in postsurgical complications such as pain, paralysis, and persistent numbness. ${ }^{1,2}$ Current techniques to avoid intraoperative nerve injury rely heavily on a surgeon's skill level and experience to visually avoid these typically small, buried structures. A contrast agent specific for nerve tissue could aid in identification and visualization of nerves during surgery, as a number of fluorescence image-guided surgical systems are either in clinical trial or have FDA approval such as the FLARE ${ }^{\mathrm{TM}}$ by Curadel, Fluorobeam by Fluoptics, da Vinci robotic systems by Intuitive Surgical, SPY by Novadaq, Photodynamic Eye by Hamamatsu, and Artemis by Quest Medical. ${ }^{3,4}$ Imaging agents that can provide tissue-specific fluorescent signal have the potential to deliver a high contrast means of visualizing target tissues using such fluorescence-guided surgical systems. However, currently there are only a handful of fluorescent contrast agents approved for clinical use including fluorescein, aminolevulinic acid-protoporphyin IX, methylene blue, and indocyanine green, none of which have any nerve specificity. ${ }^{5-9}$

A limited number of small molecule fluorescent contrast agents exist that stain nerve tissue in vivo, with six known classes of small molecule fluorophores that have been shown to have nerve or brain specificity. These include a handful of stilbene derivatives, ${ }^{10}$ a coumarin analog, ${ }^{11}$ a library of distyrylbenzene derivatives, ${ }^{12-15} 8$ styryl pyridinium (FM) fluorophores, ${ }^{16,17}$ an oxazine fluorophore, ${ }^{2,18}$ and a tricarbocyanine fluorophore. ${ }^{19}$ Unfortunately, adipose specificity often accompanies nerve specificity for these small molecule fluorophores, ${ }^{2,13,14}$ which is largely by necessity since the molecular composition of nerve and adipose tissues is similar, ${ }^{20,21}$ making specific accumulation in the nerve without adipose accumulation a substantial challenge. This dual tissue accumulation compounds the problem of identifying and visualizing nerve tissue using fluorescence, as nerves are often adjacent to or embedded within adipose tissue. Of the known small molecule nerve-specific fluorophores, oxazines have demonstrated the greatest utility since the lead compound is redshifted and peripheral nerve specificity is high. However, the current lead nerve-specific oxazine compound, oxazine 4 , does not have the desired nearinfrared (NIR) excitation or emission, ${ }^{2,18}$ making additional chemical modifications necessary to generate a clinically relevant agent.

Interestingly, the commercially available Nile Red is also an oxazine fluorophore; however, unlike oxazine 4 , it has a strong tendency to accumulate in lipid- and adipose-rich environments. ${ }^{22}$ In addition, in apolar environments such as lipids, Nile Red exhibits a high quantum yield and hypsochromatically shifted absorbance and emission spectra. In contrast, in polar environments such as water, Nile Red exhibits bathochrommatically shifted absorbance and emission spectra with a quantum yield that approaches zero. ${ }^{23}$ These spectral properties have led to a wide range of applications from evaluation of lipid droplets in tissue to its use in PEGylated nanoparticles for 
iron detection. ${ }^{24}$ More recently, Nile Red has been shown to improve nerve-specific contrast of oxazine 4 in a study that used dual fluorophore staining to enhance visualization of nerve tissue during in vivo studies where adipose, nerve, and muscle tissues were present. ${ }^{2}$ While the adipose specificity of Nile Red was high, it showed some accumulation in nerve tissue after administration, reducing the contrast enhancing benefits of its use as a ratiometric fluorophore to enhance nerve contrast.

Herein, we report the synthesis of eight Nile Red derivatives with varied electron donating and withdrawing groups to try to influence tissue-specific accumulation and generate a truly adipose-specific fluorophore. In vitro, ex vivo, and in vivo staining studies were completed to assess general lipid staining as well as nerve-, adipose-, and muscle-specific accumulation and contrast from each Nile Red derivative was compared to the parent compound. Excitingly, a Nile Red derivative, Nile Red 5 (NR5), was found that showed adipose-specific contrast and decreased nerve accumulation as compared to Nile Red. Notably, NR5 also resulted in improved adipose-to-nerve and adipose-tomuscle contrast compared to Nile Red, especially when longer time points relevant to surgical interventions were considered. Ratiometric imaging with NR5 and oxazine 4 compared to Nile Red and oxazine 4 demonstrated improved nerve contrast using NR5 as compared to Nile Red. This derivative could be used in future work to improve nerve-specific ratiometric imaging with oxazine 4 or other nerve-specific fluorophores for fluorescence image-guided surgery.

\section{Materials and Methods}

\subsection{Chemicals and Solvents}

All fluorophores, chemicals, and solvents were purchased from Sigma-Aldrich (St. Louis, Missouri) or Thermo Fisher Scientific (Waltham, Massachusetts) and used without further purification unless otherwise noted. All solvents were American Chemical Society grade unless otherwise noted. Silica gel, 230 to 400 mesh was used for all column chromatography.

\subsection{Synthetic Confirmation and Fluorophore Characterization}

Tandem liquid chromatography mass spectroscopy (LCMS) analysis (Agilent 1260 LC system with a 6224-time of flight MS, Agilent, Santa Clara, California) was performed using a $2.7-\mu \mathrm{m}$ C18 column $\left(4.6 \times 50 \mathrm{~mm}^{2}\right.$, Agilent). High-performance liquid chromatography (HPLC) purification (Agilent 1260 system with a preparative pump) was performed using a $5-\mu \mathrm{m}$ C18 column $\left(21.2 \times 150 \mathrm{~mm}^{2}\right.$, Agilent $)$. For both HPLC and LCMS, the mobile phase consisted of HPLC grade acetonitrile and water, treated with $0.1 \%$ formic acid. Spectroscopic analysis was performed on a Spectramax M5 spectrometer (Molecular Devices, Sunnyvale, California) using a quartz cuvette with a 1-cm pathlength. Quantum yield was calculated as

$\varnothing_{x}=\varnothing_{s} \times \frac{F_{x}}{F_{s}} \times \frac{f_{s}}{f_{x}} \times \frac{n_{x}}{n_{s}}$,

where $\varnothing$ is the quantum yield, $F$ is the integrated fluorescence intensity, $f$ is the absorbance at the excitation wavelength, and $n$ is the refractive index of the solvent. $S$ refers to the quantum yield standard (rhodamine $\mathrm{B})^{25}$ and $x$ refers to the unknown sample.

\subsubsection{Synthesis of fluorophore base structures}

5-(diethylamino)-2-nitrosophenol (2). $1.02 \mathrm{~g}(6.16 \mathrm{mmol})$ of 3-diethylaminophenol (1) was dissolved in $16 \mathrm{~mL}$ of $2 \mathrm{M}$ hydrochloric acid $(\mathrm{HCl})$ and cooled in an ice bath for the duration of the reaction. $466.1 \mathrm{mg}(6.76 \mathrm{mmol})$ of sodium nitrite $\left(\mathrm{NaNO}_{2}\right)$ was added dropwise, and the reaction was stirred for $2 \mathrm{~h}$ to yield the nitroso compound 2 . The nitroso compound was filtered and used as the $\mathrm{HCl}$ salt without further purification (Fig. 1). $\mathrm{ESI}^{+}$calculated for $\mathrm{C}_{10} \mathrm{H}_{15} \mathrm{~N}_{2} \mathrm{O}_{2}: 195.11 \mathrm{~m} / \mathrm{z}$; measured: $195.11 \mathrm{~m} / \mathrm{z}$.

9-(diethylamino)-2-hydroxy-5H-benzo[a]phenoxazin-5one (3). $129.6 \mathrm{mg}(0.81 \mathrm{mmol})$ of 1,6 dihydroxynapthalene and $142.9 \mathrm{mg}(0.62 \mathrm{mmol})$ of 2 were dissolved in $3 \mathrm{~mL}$ of dimethylformamide (DMF) and heated to $100^{\circ} \mathrm{C}$ under a blanket of nitrogen. The reaction was allowed to proceed for $4 \mathrm{~h}$ and was monitored via thin layer chromatography (TLC) [dichloromethane (DCM):methanol (MeOH): 95:5] (Fig. 1). The solvent was removed under reduced pressure and the product was purified by column chromatography, eluting with chloroform $\left(\mathrm{CHCl}_{3}\right)$ :methanol (MeOH):triethylamine (TEA), 100:3:2). ESI $^{+}$calculated for $\mathrm{C}_{20} \mathrm{H}_{19} \mathrm{~N}_{2} \mathrm{O}_{3}: 335.14 \mathrm{~m} / \mathrm{z}$; measured: $335.14 \mathrm{~m} / \mathrm{z}$.

9-(diethylamino)-3-hydroxy-5H-benzo[a]phenoxazin-5one (4). $196.0 \mathrm{mg}$ (1.22 mmol) of 1,7-dihyroxynaphthalene and $209 \mathrm{mg}$ ( $0.91 \mathrm{mmol})$ of compound $\mathbf{2}$ were dissolved in $3 \mathrm{~mL}$ of DMF and heated to $100^{\circ} \mathrm{C}$ under a blanket of nitrogen. The reaction was allowed to proceed for $4 \mathrm{~h}$ and was monitored via TLC (DCM:MeOH, 95:5) (Fig. 1). The solvent was removed under reduced pressure and the product was purified by column chromatography, eluting with DCM:MeOH: (100:3). $\mathrm{ESI}^{+}$calculated for $\mathrm{C}_{20} \mathrm{H}_{19} \mathrm{~N}_{2} \mathrm{O}_{3}: 335.14 \mathrm{~m} / \mathrm{z}$; measured: $335.14 \mathrm{~m} / \mathrm{z}$.

9-(diethylamino)-5-oxo-5H-benzo[a]phenoxazin-3-yl trifluoromethanesulfonate (5). $88.2 \mathrm{mg}(0.25 \mathrm{mmol})$ of
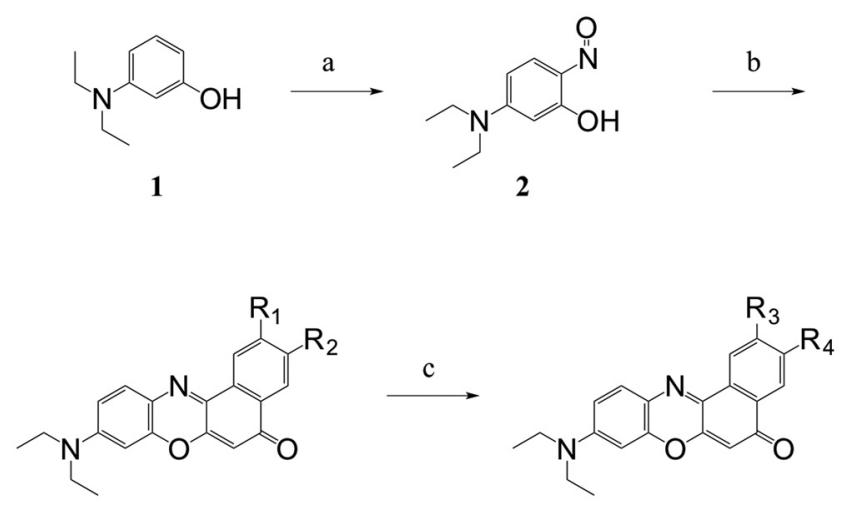

$$
\begin{aligned}
& \text { 3: } \mathrm{R}_{1}=\mathrm{OH}, \mathrm{R}_{2}=\mathrm{H} \\
& \text { 4: } \mathrm{R}_{1}=\mathrm{H}, \mathrm{R}_{2}=\mathrm{OH} \\
& \begin{array}{l}
\text { 5: } \mathrm{R}_{3}=\mathrm{CF}_{3} \mathrm{SO}_{3}, \mathrm{R}_{4}=\mathrm{H} \\
\text { 6: } \mathrm{R}_{3}=\mathrm{H}, \mathrm{R}_{2}=\mathrm{CF}_{3} \mathrm{SO}_{3}
\end{array}
\end{aligned}
$$

Fig. 1 Synthesis of fluorophore base structures. Reagents and condition: (a) $\mathrm{NaNO}_{2}, 2 \mathrm{M} \mathrm{HCl}, 0^{\circ} \mathrm{C}$ (b) 1,6/7-dihydroxynaphthalene, DMF, $100^{\circ} \mathrm{C}$, and (c) $\mathrm{Tf}_{2} \mathrm{NPh}, \mathrm{TEA}, \mathrm{DMF}, 100^{\circ} \mathrm{C}$. 
$\mathrm{N}$-phenyl-bis(trifluoromethanesulfonimide) $\quad\left(\mathrm{Tf}_{2} \mathrm{NPh}\right) \quad$ and $32 \mathrm{mg}(0.10 \mathrm{mmol})$ of 3 were added to a scintillation vial and flushed with nitrogen. Anhydrous tetrahydrofuran (THF, $3 \mathrm{~mL})$ followed by $400 \mu \mathrm{L}(2.86 \mathrm{mmol})$ of TEA were added to the reaction vessel and it was tightly capped. The reaction was carried out overnight at room temperature. Solvent was removed under reduced pressure and the product was purified by column chromatography, eluting with $\mathrm{CHCl}_{3}: \mathrm{MeOH}(100: 3)$ (Fig. 1). $\mathrm{ESI}^{+}$calculated for $\mathrm{C}_{21} \mathrm{H}_{18} \mathrm{~F}_{3} \mathrm{~N}_{2} \mathrm{O}_{5} \mathrm{~S}: 467.09 \mathrm{~m} / \mathrm{z}$; measured: $467.09 \mathrm{~m} / \mathrm{z}$.

9-(diethylamino)-5-oxo-5H-benzo[a]phenoxazin-4-yl trifluoromethanesulfonate (6). $64.8 \mathrm{mg}(0.181 \mathrm{mmol})$ of $\mathrm{Tf}_{2} \mathrm{NPh}$ and $23.8 \mathrm{mg}(0.0712 \mathrm{mmol})$ of 4 were added to a scintillation vial and flushed with nitrogen. Anhydrous THF (3 mL) and $284 \mu \mathrm{L}$ ( $2.04 \mathrm{mmol})$ of TEA were added to the reaction vessel and it was tightly capped. The reaction was carried out overnight at room temperature. The solvent was removed under reduced pressure and the product was purified by column chromatography, eluting with $\mathrm{CHCl}_{3}$ : $\mathrm{MeOH}$ (100:3) (Fig. 1). $\mathrm{ESI}^{+}$calculated for $\mathrm{C}_{21} \mathrm{H}_{18} \mathrm{~F}_{3} \mathrm{~N}_{2} \mathrm{O}_{5} \mathrm{~S}: 467.09 \mathrm{~m} / \mathrm{z}$; measured: $467.09 \mathrm{~m} / \mathrm{z}$.

\subsubsection{Sonogashira Nile Red derivative synthesis}

4-([9-(diethylamino)-5-oxo-5H-benzo[a]phenoxazin-2yl]ethynyl)-2-(methylsulfonyl)benzaldehyde [Nile Red 6 (NR6)]. $\quad 1.6 \mathrm{mg}(0.009 \mathrm{mmol})$ of palladium(II) chloride $\left(\mathrm{PdCl}_{2}\right), 1.8 \mathrm{mg}(0.0095 \mathrm{mmol})$ of copper(I) iodide $(\mathrm{CuI})$, and $2.3 \mathrm{mg}(0.0088 \mathrm{mmol})$ of triphenylphosphine $\left(\mathrm{PPh}_{3}\right)$ were added to a round-bottom flask. All solvents were sparged with nitrogen prior to use and the reaction was performed under a blanket of nitrogen. $6.1 \mathrm{mg}(0.013 \mathrm{mmol})$ of 5 was dissolved in DMF $(2 \mathrm{~mL})$ and added to the flask followed by $53 \mu \mathrm{L}$ $(0.3803 \mathrm{mmol})$ of TEA. Trimethylsilylacetylene (TMS acetylene) was cooled in an ice bath and $53 \mu \mathrm{L}(0.3826 \mathrm{mmol})$ were added to the reaction mixture. The reaction was run overnight at room temperature. The following morning, $23.6 \mu \mathrm{L}$ $(0.156 \mathrm{mmol})$ of 1,8-diazabicyclo[5.4.0]undec-7-ene (DBU), $0.1 \mu \mathrm{L}(0.006 \mathrm{mmol})$ of water, and $11.4 \mathrm{mg}(0.043 \mathrm{mmol})$ of 4-bromo-2-(methylsulfonyl)benzaldehyde were added to the reaction, and it was heated to $70^{\circ} \mathrm{C}$ for $24 \mathrm{~h}$ (Fig. 2). The final compound was purified via HPLC with a water:acetonitrile gradient of 90:10 to $10: 90$ over $20 \mathrm{~min}$. $\mathrm{ESI}^{+}$calculated for $\mathrm{C}_{30} \mathrm{H}_{25} \mathrm{~N}_{2} \mathrm{O}_{5} \mathrm{~S}^{+}$: $525.15 \mathrm{~m} / \mathrm{z}$; measured: $525.15 \mathrm{~m} / \mathrm{z}$.
2-[(3-aminophenyl)ethynyl]-9-(diethylamino)-5H-benzo [a]phenoxazin-5-one [(Nile Red 7 (NR7)]. $0.8 \mathrm{mg}$ $(0.0045 \mathrm{mmol})$ of $\mathrm{PdCl}_{2}, 1.9 \mathrm{mg}(0.01 \mathrm{mmol})$ of $\mathrm{CuI}$, and $2.4 \mathrm{mg}(0.0092 \mathrm{mmol})$ of $\mathrm{PPh}_{3}$ were added to a round-bottom flask. All solvents were sparged with nitrogen prior to use and the reaction was performed under a blanket of nitrogen. $7.8 \mathrm{mg}$ $(0.0167 \mathrm{mmol})$ of $\mathbf{5}$ were dissolved in DMF $(2 \mathrm{~mL})$ and added to the flask followed by $53 \mu \mathrm{L}(0.3803 \mathrm{mmol})$ of TEA. TMS acetylene was cooled in an ice bath and $53 \mu \mathrm{L}(0.3826 \mathrm{mmol})$ were added to the reaction mixture. The reaction was run overnight at room temperature. The following morning $30 \mu \mathrm{L}(0.20 \mathrm{mmol})$ of $\mathrm{DBU}, 0.120 \mu \mathrm{L}(0.007 \mathrm{mmol})$ of water, and $1.82 \mu \mathrm{L}$ $(0.167 \mathrm{mmol})$ of 3 -bromoaniline were added to the reaction, and it was heated to $70^{\circ} \mathrm{C}$ for $3 \mathrm{~h}$ (Fig. 2). The final compound was purified via HPLC with a water:acetonitrile gradient of 90:10 to 10:90 over 20 min. $\mathrm{ESI}^{+}$calculated for $\mathrm{C}_{29} \mathrm{H}_{23} \mathrm{~N}_{2} \mathrm{O}_{3}^{+}$: $434.19 \mathrm{~m} / \mathrm{z}$; measured: $434.19 \mathrm{~m} / \mathrm{z}$.

3-([9-(diethylamino)-5-oxo-5H-benzo[a]phenoxazin-2yl]ethynyl)benzaldehyde [Nile Red 8 (NR8)]. $1.0 \mathrm{mg}$ $(0.0056 \mathrm{mmol})$ of $\mathrm{PdCl}_{2}, 1.2 \mathrm{mg}(0.0063 \mathrm{mmol})$ of $\mathrm{CuI}$, and $2.7 \mathrm{mg}(0.0103 \mathrm{mmol})$ of $\mathrm{PPh}_{3}$ were added to a round-bottom flask. All solvents were sparged with nitrogen prior to use and the reaction was performed under a blanket of nitrogen. $11.2 \mathrm{mg}$ $(0.024 \mathrm{mmol})$ of $\mathbf{5}$ were dissolved in DMF $(2 \mathrm{~mL})$ and added to the flask followed by $33 \mu \mathrm{L}(0.2368 \mathrm{mmol})$ of TEA. TMS acetylene was cooled in an ice bath and $33 \mu \mathrm{L}(0.2382 \mathrm{mmol})$ were added to the reaction mixture. The reaction was run overnight at room temperature. The following morning $43 \mu \mathrm{L}(0.288 \mathrm{mmol})$ of DBU, $0.172 \mu \mathrm{L}(0.0096 \mathrm{mmol})$ of water, and $5.8 \mathrm{mg}$ $(0.025 \mathrm{mmol})$ of 3 -iodobenzaldehyde were added to the reaction, and it was heated to $75^{\circ} \mathrm{C}$ for $3 \mathrm{~h}$ (Fig. 2). The final compound was purified via HPLC with a water:acetonitrile gradient of 90:10 to 10:90 over $20 \mathrm{~min}$. $\mathrm{ESI}^{+}$calculated for $\mathrm{C}_{29} \mathrm{H}_{23} \mathrm{~N}_{2} \mathrm{O}_{3}^{+}$: $447.17 \mathrm{~m} / \mathrm{z}$; measured: $447.17 \mathrm{~m} / \mathrm{z}$.

3-([9-(diethylamino)-5-oxo-5H-benzo[a]phenoxazin-2yl]ethynyl)-4-methoxybenzaldehyde [Nile Red 9 (NR9)]. $\quad 1.4 \mathrm{mg}(0.008 \mathrm{mmol})$ of $\mathrm{PdCl}_{2}, 1.7 \mathrm{mg}$ $(0.0089 \mathrm{mmol})$ of $\mathrm{CuI}$, and $2.3 \mathrm{mg}(0.0088 \mathrm{mmol})$ of $\mathrm{PPh}_{3}$ were added to a round-bottom flask. All solvents were sparged with nitrogen prior to use and the reaction was performed under a blanket of nitrogen. $13 \mathrm{mg}(0.028 \mathrm{mmol})$ of 5 were dissolved in DMF $(1 \mathrm{~mL})$ and added to the flask followed by $53 \mu \mathrm{L}$ $(0.3803 \mathrm{mmol})$ of TEA. TMS acetylene was cooled in an ice

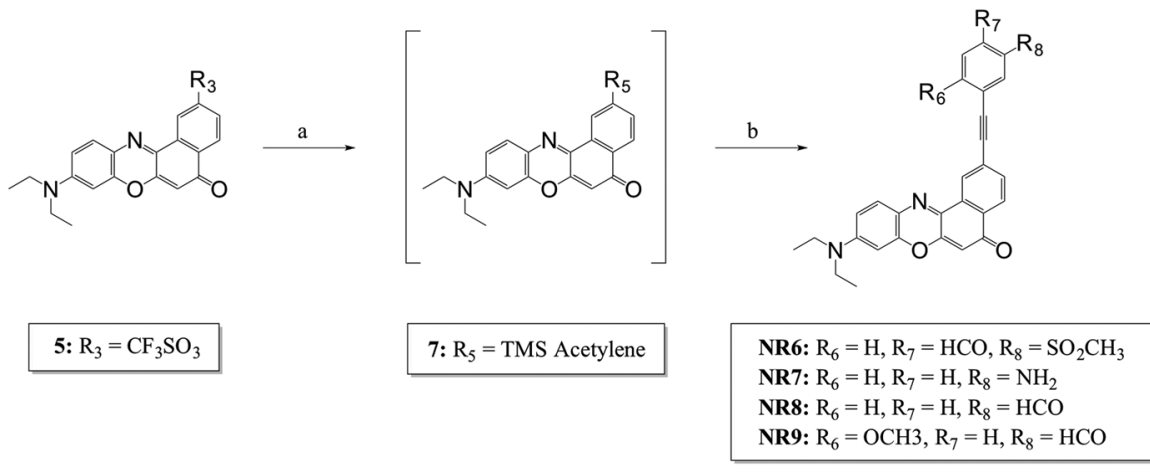

Fig. 2 Sonogashira Nile Red derivative synthesis. Reagents and conditions: (a) TMS acetylene, $\mathrm{Pd}(0)$, Cul, $\mathrm{PPh}_{3}$, TEA, DMF, RT, overnight and (b) DBU, $\mathrm{H}_{2} \mathrm{O}$, aryl halide, DMF, RT, overnight. 
bath and $53 \mu \mathrm{L}(0.3826 \mathrm{mmol})$ were added to the reaction mixture. The reaction was run overnight at room temperature. The following morning $50.25 \mu \mathrm{L}(0.336 \mathrm{mmol})$ of DBU, $0.2 \mu \mathrm{L}$ $(0.011 \mathrm{mmol})$ of water, and $8.4 \mathrm{mg}(0.039 \mathrm{mmol})$ of $4-$ bromo-2-(methylsulfonyl)benzaldehyde were added to the reaction, and it was heated to $70^{\circ} \mathrm{C}$ for $24 \mathrm{~h}$ (Fig. 2). The final compound was purified via HPLC with a water:acetonitrile gradient of $90: 10$ to $10: 90$ over $20 \mathrm{~min}$. $\mathrm{ESI}^{+}$calculated for $\mathrm{C}_{30} \mathrm{H}_{25} \mathrm{~N}_{2} \mathrm{O}_{4}^{+}$: $477.18 \mathrm{~m} / \mathrm{z}$; measured: $477.18 \mathrm{~m} / \mathrm{z}$.

\subsubsection{Suzuki Nile Red derivative synthesis}

3-(4-aminophenyl)-9-(diethylamino)-5H-benzo[a]phenoxazin-5-one [Nile Red 1 (NRI)]. $14.5 \mathrm{mg}(0.031 \mathrm{mmol})$ of $6,0.3 \mathrm{mg}(0.0013 \mathrm{mmol})$ of palladium (II) acetate $\left[\mathrm{Pd}(\mathrm{OAc})_{2}\right], \quad 0.6 \mathrm{mg}(0.0023 \mathrm{mmol})$ of $\mathrm{PPh}_{3}, 5.8 \mathrm{mg}$ $(0.1 \mathrm{mmol})$ of potassium fluoride $(\mathrm{KF})$, and $7.8 \mathrm{mg}$ (0.036 mmol) of 4-aminophenylboronic acid pinacol ester were added to a round-bottom flask and subsequently flushed with nitrogen. $1 \mathrm{~mL}$ of DMF was degassed under vacuum with sonication and added to the reaction vessel. The reaction was run at $70^{\circ} \mathrm{C}$ overnight (Fig. 3). The final compound was purified via HPLC with a water:acetonitrile gradient of 90:10 to $10: 90$ over $20 \mathrm{~min}$. $\mathrm{ESI}^{+}$calculated for $\mathrm{C}_{26} \mathrm{H}_{24} \mathrm{~N}_{3} \mathrm{O}_{2}^{+}$: $410.19 \mathrm{~m} / \mathrm{z}$; measured: $410.19 \mathrm{~m} / \mathrm{z}$.

4-[9-(diethylamino)-5-oxo-5H-benzo[a]phenoxazin-3-yl] benzonitrile [Nile Red 5 (NR5)]. $14.5 \mathrm{mg}(0.031 \mathrm{mmol})$ of 6, $0.3 \mathrm{mg}(0.0013 \mathrm{mmol})$ of $\mathrm{Pd}(\mathrm{OAc})_{2}, 0.6 \mathrm{mg}(0.0023 \mathrm{mmol})$ of $\mathrm{PPh}_{3}, 5.8 \mathrm{mg}(0.1 \mathrm{mmol})$ of $\mathrm{KF}$, and $6.4 \mathrm{mg}(0.044 \mathrm{mmol})$ of 4-cyanophenylboronic acid were added to a round-bottom flask and subsequently flushed with nitrogen. $1 \mathrm{~mL}$ of DMF was degassed under vacuum with sonication and added to the reaction vessel. The reaction was run at $70^{\circ} \mathrm{C}$ overnight (Fig. 3). The final compound was purified via HPLC with a water:acetonitrile gradient of 90:10 to 10:90 over $20 \mathrm{~min}$. $\mathrm{ESI}^{+}$calculated for $\mathrm{C}_{27} \mathrm{H}_{22} \mathrm{~N}_{3} \mathrm{O}_{2}^{+}$: $420.17 \mathrm{~m} / \mathrm{z}$; measured: $420.17 \mathrm{~m} / \mathrm{z}$.

2-(4-aminophenyl)-9-(diethylamino)-5H-benzo[a]phenoxazin-5-one [Nile Red $10 \quad$ (NR10)]. $8.0 \quad \mathrm{mg}$ $(0.017 \mathrm{mmol})$ of $5,0.3 \mathrm{mg}(0.0013 \mathrm{mmol})$ of $\mathrm{Pd}(\mathrm{OAc})_{2}$, $0.5 \mathrm{mg}(0.0019 \mathrm{mmol})$ of $\mathrm{PPh}_{3}, 3.1 \mathrm{mg}(0.053 \mathrm{mmol})$ of $\mathrm{KF}$, and $5.0 \mathrm{mg}(0.023 \mathrm{mmol})$ of 4-aminophenylbornic acid pinacol ester were added to a round-bottom flask. The flask was flushed with nitrogen and nitrogen-sparged THF $(430 \mu \mathrm{L})$ was added. The reaction was run at $60^{\circ} \mathrm{C}$ overnight (Fig. 3). The final compound was purified via HPLC with a water:acetonitrile gradient of 90:10 to 10:90 over $20 \mathrm{~min}$. $\mathrm{ESI}^{+}$calculated for $\mathrm{C}_{26} \mathrm{H}_{24} \mathrm{~N}_{3} \mathrm{O}_{2}^{+}$: $410.19 \mathrm{~m} / \mathrm{z}$; measured: $410.19 \mathrm{~m} / \mathrm{z}$.

4-[9-(diethylamino)-5-oxo-5H-benzo[a]phenoxazin-2-yl] benzonitrile [Nile Red 12 (NR12)]. $7.4 \mathrm{mg}(0.022 \mathrm{mmol})$ of 5, $0.2 \mathrm{mg}(0.0011)$ of $\mathrm{PdCl}_{2}, 0.7 \mathrm{mg}(0.0027)$ of $\mathrm{PPh}_{3}$, and $3.9 \mathrm{mg}(0.0265 \mathrm{mmol})$ of 4-cyanophenylboronic acid were added to a round-bottom flask. The flask was flushed with nitrogen and $200 \mu \mathrm{L}$ of nitrogen sparged THF was added. A 2 M solution of potassium carbonate was sparged with nitrogen and $64.5 \mu \mathrm{L}$ was added to the reaction mixture. The reaction was run at $60^{\circ} \mathrm{C}$ overnight (Fig. 3). The final compound was purified via HPLC with a water:acetonitrile gradient of 90:10 to 10:90 over $20 \mathrm{~min}$. $\mathrm{ESI}^{+}$calculated for $\mathrm{C}_{27} \mathrm{H}_{22} \mathrm{~N}_{3} \mathrm{O}_{2}$ : $420.17 \mathrm{~m} / \mathrm{z}$; measured: $420.17 \mathrm{~m} / \mathrm{z}$.

\subsection{Cell Culture for Nile Red Derivative Staining}

The U2OS human osteosarcoma cell line was cultured in Dulbecco's modified Eagle medium (DMEM, Thermo Fisher Scientific) with $10 \%$ fetal bovine serum (FBS, VWR, Radnor, Pennysylvania) and $1 \%$ penicillin/streptomycin 100X (Thermo Fisher Scientific) at $37^{\circ} \mathrm{C}$ in $5 \% \mathrm{CO}_{2}$. For live cell imaging studies, FluoroBrite DMEM (Thermo Fisher Scientific) was used in place of DMEM. For imaging studies, cells were grown in 96-well glass bottom plates (Cellvis, Mountain View, California) for $48 \mathrm{~h}$, with a final confluence of $<80 \%$. Nile Red and its derivatives were prepared at 1 to $10 \mathrm{mM}$ stock concentration in dimethyl sulfoxide (DMSO) and diluted into media or buffer for cell staining and imaging studies.

\subsection{Nile Red Derivative Cell Staining and Imaging Studies}

Cell staining studies were completed on fixed and live U2OS cells using Nile Red and each synthesized Nile Red derivative described as follows. For fixed cell staining, cells were washed once with $1 \times$ phosphate-buffered saline (PBS), then fixed with $4 \%$ paraformaldehyde (PFA) for $20 \mathrm{~min}$ at room temperature. The PFA was removed and each well was washed three times for 5 min per wash $(3 \times 5$ mins $)$ with $1 \times$ PBS. The Nile Red fluorophores were diluted into $1 \times$ PBS to a final concentration of $1 \mu \mathrm{M}$, incubated for $5 \mathrm{~min}$ with the cells, and images were collected without washing. Live cell staining was completed using two methods. First, cells were washed once with FluoroBrite DMEM. The Nile Red fluorophore solution was prepared in FluoroBrite DMEM by diluting to a final concentration of $1 \mu \mathrm{M}$ for staining, where cells were stained for $5 \mathrm{~min}$.

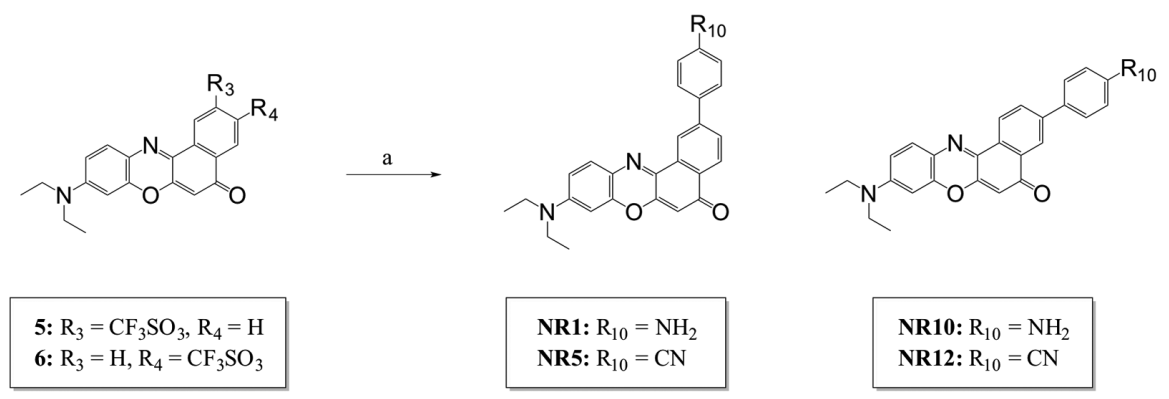

Fig. 3 Suzuki Nile Red derivative synthesis. Reagents and conditions: (a) boronic acid, base, $\mathrm{Pd}(0)$, $\mathrm{PPh}_{3}, \mathrm{DMF}, 60^{\circ} \mathrm{C}$ to $70^{\circ} \mathrm{C}$. 
The fluorophore-containing media were removed and the cells were fixed for 20 min using 4\% PFA. Cells were then washed $3 \times 5$ min with FluoroBrite DMEM prior to imaging. Second, cells were washed with FluoroBrite DMEM without FBS once for $5 \mathrm{~min}$. Nile Red fluorophore solutions were prepared in FluoroBrite without FBS at $1 \mu \mathrm{M}$ final concentration for cell staining. The cells were stained with the prepared solution for $5 \mathrm{~min}$ and imaged in the staining solution. All images were collected at their ideal exposure times to permit visualization of stained intracellular structures.

\subsection{Nile Red Derivative Ex Vivo Tissue Staining, Imaging, and Fluorescence Quantification}

Cryosections of CD-1 mouse sciatic nerve and surrounding muscle and adipose tissues from unrelated experiments were cut from optimal cutting temperature (OCT, Thermo Fisher Scientific) embedded frozen tissues. Cryosectioned tissues were captured on superfrost plus slides (Thermo Fisher Scientific) at $10 \mu \mathrm{m}$ thickness. The tissue sections were washed with $1 \times$ PBS for 2 min to remove OCT, and then fixed with $2 \%$ PFA for $15 \mathrm{~min}$. The tissue sections were then washed with $0.1 \%$ Tween 20 in $1 \times$ PBS $(3 \times 5 \mathrm{~min})$, followed by incubation with $100 \mu \mathrm{M}$ of Nile Red and each Nile Red derivative formulated using 10\% DMSO, 5\% Kolliphore (Sigma-Aldrich), $63.75 \%$ FBS, and $21.25 \% 1 \times$ PBS as previously described. ${ }^{26}$ Tissue sections were incubated with the formulated fluorophores for $40 \mathrm{~min}$ at room temperature, followed by washing with blank formulation not containing fluorophore $(2 \times 5 \mathrm{~min})$. Each tissue section was then washed with $1 \times$ PBS $(2 \times 5 \mathrm{~min})$, fixed for 15 min using $2 \%$ PFA, followed by a final wash with $1 \times$ PBS for 5 min before mounting with glycerol (Thermo Fisher Scientific). Images were collected at an exposure time of $650 \mathrm{~ms}$ for all Nile Red and Nile Red derivative-stained sections. Four tissue sections were stained for Nile Red and each Nile Red derivative, facilitating adipose-to-muscle and adiposeto-nerve contrast ratio quantification where tissue intensities were quantified using region of interest analysis with custom written MatLab code (The MathWorks, Natick, Massachusetts). For each tissue type, a representative area was selected, where a threshold-based mask was set to determine tissue from background. The mean tissue intensity was calculated in adipose, nerve, and muscle tissues as the intensity per second and used to calculate the adipose-to-nerve and adipose-to-muscle contrast ratios.

\subsection{Fluorescence Microscopy}

All ex vivo and cellular fluorescence and white light images were collected using a Zeiss AxioObserver inverted fluorescence microscope (Carl Zeiss, Oberkochen, Germany). All cell images were collected at $40 \times$ magnification and all tissue section images were collected at $10 \times$ magnification. Fluorescence excitation was completed using a Photofluor II broadband light source (Burlington, Vermont) filtered with a $545 \pm 12.5$-nm bandpass excitation filter. Fluorescence images were collected using a $605 \pm 35$-nm bandpass emission filter and an AxioCam 506 monochrome camera (Zeiss). All filters were purchased from Chroma Technologies (Bellows Falls, Vermont).

\subsection{In Vivo Imaging Following Direct/Topical Administration in Rodent Nerves}

In vivo images were acquired using a previously published, custom-built small animal fluorescence imaging system, ${ }^{27}$ described briefly here. Fluorescence images were obtained using a Qimaging EXi Blue monochrome camera (Surrey, British Columbia, Canada) with a removable Bayer mask for collection of coregistered color and fluorescence images. A Photofluor II broadband light source was expanded onto the surgical field through a liquid light guide and used unfiltered for white light illumination. Nile Red and its derivatives were excited using a $545 \pm 12.5$-nm bandpass filter, while fluorescence emission images were collected with a $605 \pm 35 \mathrm{~nm}$ bandpass filter. Oxazine 4 (Exciton, West Chester, Ohio) was used alone as well as for nerve and adipose costaining and ratiometric imaging studies. Oxazine 4 was excited using a $620 \pm 30 \mathrm{~nm}$-bandpass filter, while fluorescence emission images were collected through a $700 \pm 37.5$-nm bandpass filter.

Approval for the use of animals in the study was obtained from the Institutional Animal Care and Use Committee at Oregon Health and Science University. In vivo staining studies were carried out on male CD-1 mice using an optimized direct/ topical administration protocol. ${ }^{2}$ Staining was performed concurrently on no more than two nerve sites (sciatic nerve or brachial plexus) per mouse. Nile Red and all Nile Red derivatives were formulated in a previously published cosolvent formulation (10\% DMSO, 5\% Kolliphor, $63.75 \%$ serum, and $21.25 \% \mathrm{PBS}^{14}$ ) at a final concentration of $100 \mu \mathrm{M}$. Each formulated fluorophore was incubated for $5 \mathrm{~min}$ on the nerve site ( $n=4$ nerve sites/fluorophore), followed by nine short flushes with PBS. The nerve site was then incubated with blank cosolvent not containing fluorophore for $5 \mathrm{~min}$, followed again by nine short flushes with PBS.

In vivo costaining was completed with (1) Nile Red and oxazine 4 as well as (2) NR5 and oxazine 4 . For these studies, cosolvent-formulated oxazine $4(125 \mu \mathrm{M})$ was incubated on the nerve site for 5 min followed by nine short flushes with PBS. The cosolvent-formulated Nile Red or NR5 $(100 \mu \mathrm{M})$ was then incubated on the nerve site for $5 \mathrm{~min}$ and also followed by nine short flushes with PBS. Coregistered (1) Nile Red and oxazine 4 or (2) NR5 and oxazine 4 images were collected from $n=4$ nerve sites per groups (1) and (2). For comparison, oxazine 4 $(125 \mu \mathrm{M})$ only in vivo staining using the optimized direct administration protocol was also completed on $n=4$ nerve sites.

\subsection{Ratiometric Image Calculation and Statistical Analysis}

Ratiometric images were calculated and quantified using region of interest analysis on the white light images to identify the nerve, adipose, and muscle tissue types. Due to the known cross talk of oxazine 4 into the Nile Red channel, ${ }^{2}$ the oxazine 4 image was subtracted from the Nile Red or NR5 image resulting in an image of only adipose. The ratiometric images were then calculated as the oxazine 4 image minus the corrected Nile Red or NR5 image divided by the corrected Nile Red or NR5 image. Oxazine 4 only images were generated from single fluorophore staining and were not ratiometric in nature. Receiver operator characteristic (ROC) curve analysis was performed to assess differences among the ratiometric images, where the area under the curve (AUC) was used as the metric to differentiate between groups (1) and (2) using custom written MatLab 
code. ${ }^{28}$ Oxazine 4 only images were also compared to images from groups (1) and (2) to assess contrast improvement using ratiometric imaging. Statistical significance between the three staining conditions was determined using the method described by Hanley and $\mathrm{McNeil}^{29}$ with standard error measurements and correlation coefficients for pixel intensity values in each channel used for $z$-score value calculations. ${ }^{28} \mathrm{~A} z$-score $>2$ indicated that the populations were statistically different with $95 \%$ confidence, while a $z$-score $>3$ indicated that the populations were statistically different with $99.7 \%$ confidence. $^{29}$

\section{Results}

\subsection{Synthesis of the Nile Red Core Derivatives}

The syntheses of the Nile Red core structures were completed in accordance with previously described methods (Fig. 1). ${ }^{30}$ The nitroso compound 2 was prepared by reacting 3-diethylaminophenol with sodium nitrite on ice in $\mathrm{HCl}$ for $2 \mathrm{~h}$. The product was filtered and used immediately without further purification. Syntheses of compounds $\mathbf{3}$ and $\mathbf{4}$ were completed by condensation of $\mathbf{2}$ and either 1,6-dihydroxynapthalene or 1,7dihydroxynapthalene at $100^{\circ} \mathrm{C}$. The resulting 2-hydroxy or 3hydroxy Nile Red derivatives were converted to their triflated versions (compounds 5 and 6) via reaction with $n$-phenyl bis(trifluoromethanesulfonimide) at room temperature. Compounds 5 and $\mathbf{6}$ were readily purified using column chromatography $\left(\mathrm{CH}_{3} \mathrm{Cl}: \mathrm{MeOH}, 100: 3\right)$.

Nile Red derivatives NR6, NR7, NR8, and NR9 were prepared using a one pot Sonogashira reaction (Fig. 2). ${ }^{31}$ Sparging with nitrogen was sufficient to remove dissolved oxygen from the solvent, and the reactions were carried out overnight with a catalytic amount of palladium, $\mathrm{PPh}_{3}$, and $\mathrm{CuI}$, in the presence of excess TEA. To induce the addition of the target aryl group, the aryl halide was added with catalytic water and excess DBU. The reaction was heated to $70^{\circ} \mathrm{C}$ and run for 3 to $24 \mathrm{~h}$.

Nile Red derivatives NR1, NR5, NR10, and NR12 were prepared using a Suzuki coupling reaction (Fig. 3) ${ }^{32,33}$ Rigorous degassing with sonication and house vacuum prior to use of any solvents yielded the most reliable reactions, as reagents were oxygen sensitive. Boronic acids, KF, the triflated Nile Red compounds, a catalytic amount of $\mathrm{Pd}(\mathrm{OAc})_{2}$, and $\mathrm{PPh}_{3}$ were added to the reaction vessel under nitrogen. The reactions were heated to $60^{\circ} \mathrm{C}$ to $70^{\circ} \mathrm{C}$ and run overnight.

\subsection{Photophysical Properties of Nile Red Derivatives}

The resulting eight Nile Red derivatives all exhibited similar absorption and emission characteristics to one another as well as to Nile Red $\left(\lambda_{\max A B S}=550 \mathrm{~nm}, \lambda_{\max \mathrm{EM}}=630 \mathrm{~nm}\right.$ in DMSO). Spectral evaluation in DMSO displayed absorbance maxima from all eight Nile Red derivatives between 555 and $570 \mathrm{~nm}$, while the emission maxima ranged from 635 to 640 nm [Fig. 4(a)]. Nile Red has well-characterized solvochromatic behavior due to a twisted intramolecular charge transfer state formed by the rotation of the diethyl amino group in the excited state. Rotation about this bond only occurs in polar solvents where it is stabilized, resulting in a bathochromic shift. $^{23,34}$ As expected, all synthesized Nile Red derivatives demonstrated similar solvochromatic behavior to Nile Red $\left(\lambda_{\max \mathrm{ABS}}=520 \mathrm{~nm}, \lambda_{\max \mathrm{EM}}=570 \mathrm{~nm}\right.$ in toluene $)$ when spectrally evaluated in toluene, with absorbance maxima between 520 and $545 \mathrm{~nm}$ and emission maxima ranging from 575 to $590 \mathrm{~nm}$ [Fig. 4(b)]. The quantum yield was determined for all synthesized derivatives in DMSO and varied widely across the Nile Red derivatives ranging from 0.04 to 0.56 (Table 1). Notably, derivatives with an electron withdrawing group (NR5, NR6, NR8, NR9, and NR12) were significantly brighter than those with electron donating groups (NR1, NR7, and

Table 1 Optical properties of Nile Red derivatives in DMSO.

\begin{tabular}{lcccc}
\hline $\begin{array}{l}\text { NR } \\
\text { Derivative }\end{array}$ & $\begin{array}{c}\text { Max Abs } \\
(\mathrm{nm})\end{array}$ & $\begin{array}{c}\text { Max Em } \\
(\mathrm{nm})\end{array}$ & $\begin{array}{c}\text { Stokes Shift } \\
(\mathrm{nm})\end{array}$ & QY \\
\hline NR1 & 555 & 635 & 80 & 0.08 \\
NR5 & 560 & 640 & 80 & 0.54 \\
NR6 & 570 & 640 & 70 & 0.45 \\
NR7 & 565 & 640 & 75 & 0.14 \\
NR8 & 565 & 640 & 75 & 0.48 \\
NR9 & 565 & 640 & 75 & 0.48 \\
NR10 & 555 & 635 & 80 & 0.04 \\
NR12 & 560 & 635 & 75 & 0.56 \\
\hline
\end{tabular}
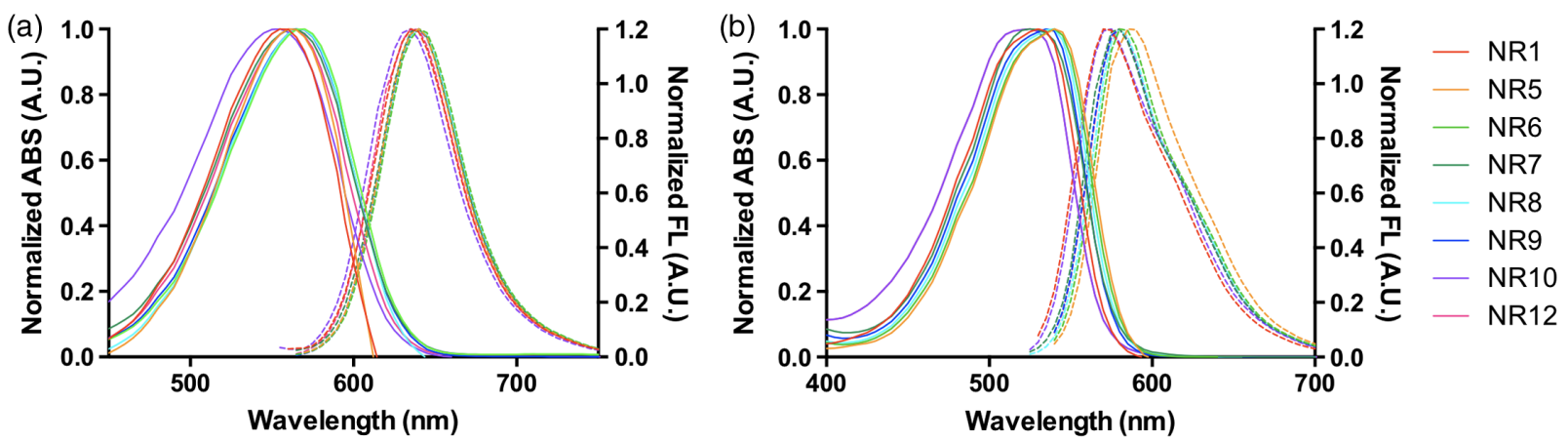

Fig. 4 Nile Red derivative absorbance and emission spectra. The normalized absorbance (solid lines) and emission (dashed lines) of NR1, NR5, NR6, NR7, NR8, NR9, NR10, and NR12 are shown in (a) DMSO and (b) toluene. 
NR12), where derivatives with electron withdrawing groups had quantum yields $>0.45$, while derivatives with electron donating groups had quantum yields $<0.14$.

\subsection{Nile Red Derivative Cell Staining and Imaging Studies}

To assess the effect of the Nile Red derivative structural diversity on lipid accumulation, fixed and live cells were stained with Nile Red and the synthesized derivatives (Fig. 5). In fixed cells, the fluorescence intensity was bright for Nile Red as well as NR5 and NR8, where both derivatives contained electron withdrawing groups and had high quantum yields. Interestingly, quantum yield was not directly correlated to in vitro fluorescence intensity since NR1 had a relatively low quantum yield $(\mathrm{QY}=0.08)$ but showed brighter in vitro fluorescence than $\mathrm{NR} 12(\mathrm{QY}=0.56)$ as determined by the optimal exposure times used for image collection [Fig. 5(a)]. As expected, lipid droplets were the prominently visible in vitro structure with Nile Red as well as its derivatives.

Fluorescence staining patterns of Nile Red and the synthesized derivatives were also assessed in cells that were stained live, then fixed [Fig. 5(b)]. Fluorescence intensity was substantially decreased, as determined from the long exposure times,
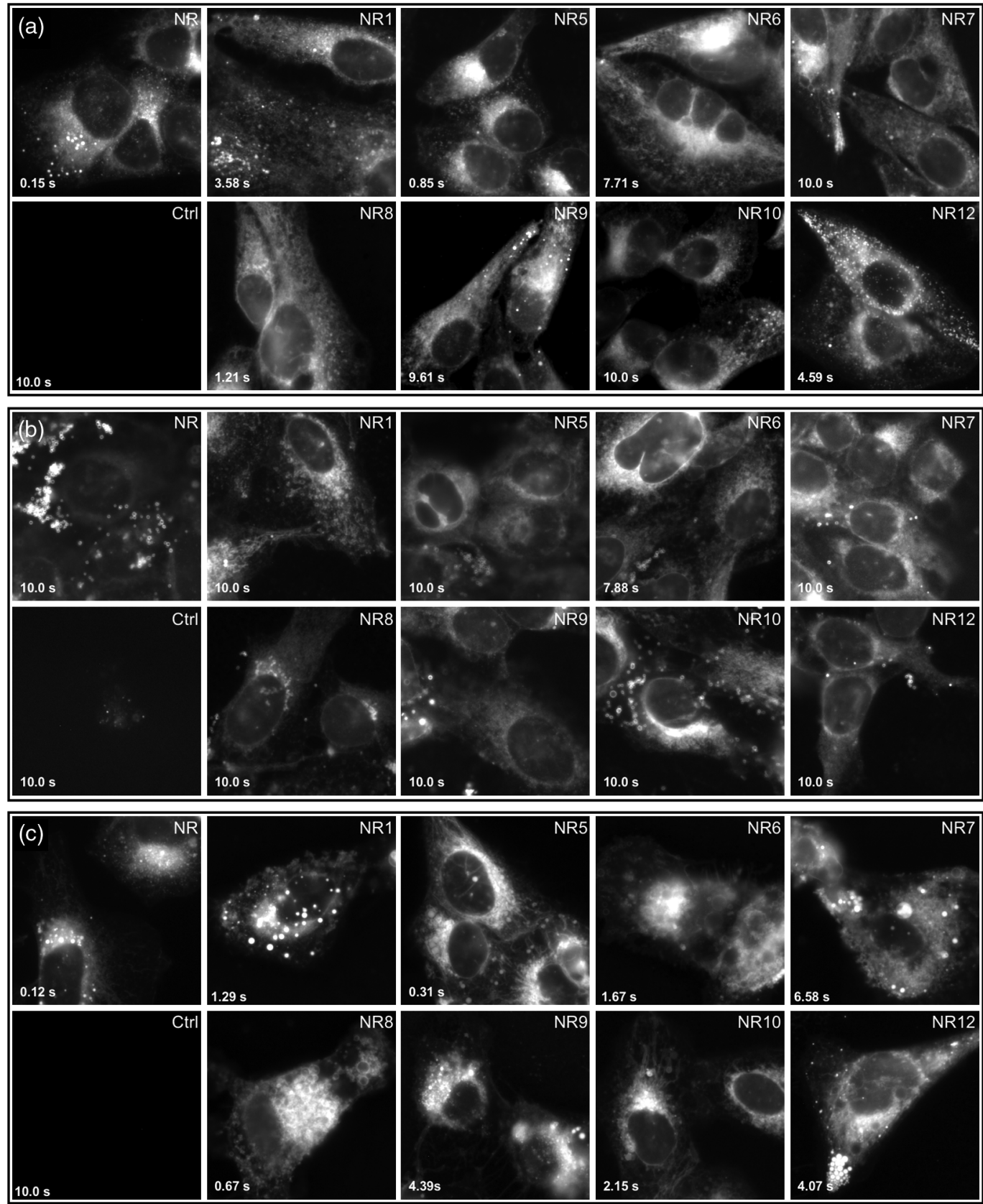

Fig. 5 In vitro Nile Red and derivative staining patterns. Nile Red and its derivatives were used to stain U2OS cells that were (a) fixed and imaged without washing, (b) live and then fixed and washed prior to imaging, and (c) live and imaged without washing. Each image was collected at the optimal exposure time and is displayed at ideal contrast to show the visible highlighted intracellular structures using Nile Red and each of its synthesized derivatives. 
which was likely due to the extra washing steps used during staining. NR6 was the only derivative to have an exposure time $<10 \mathrm{~s}$, suggesting it bound more strongly to subcellular structures than Nile Red or the other Nile Red derivatives. Overall, fluorescent staining of lipid droplet-like structures appeared diminished and more cytoplasmic structures were stained when washing was completed prior to imaging. This was particularly evident for NR6, NR7, and NR10.

Finally, the fluorescence staining patterns of Nile Red and the synthesized derivatives were assessed in live cells without (a)
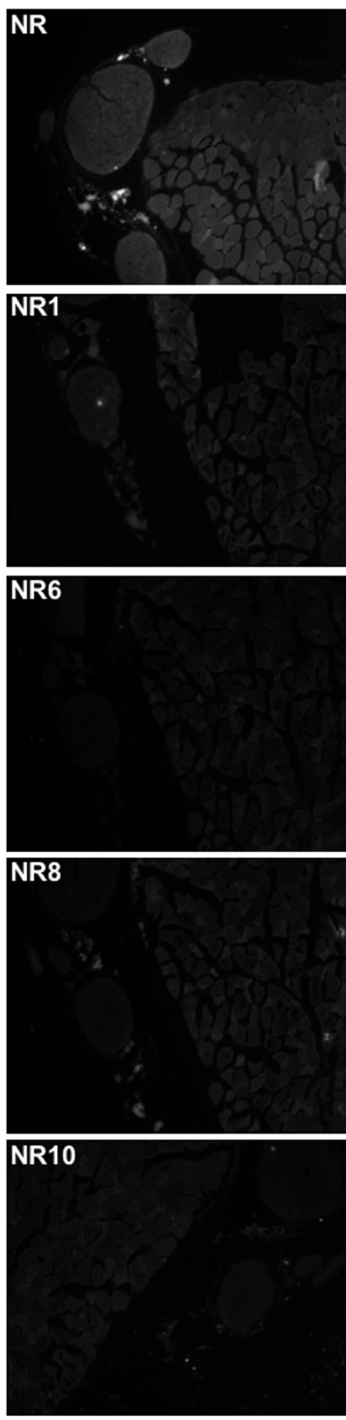

(c)
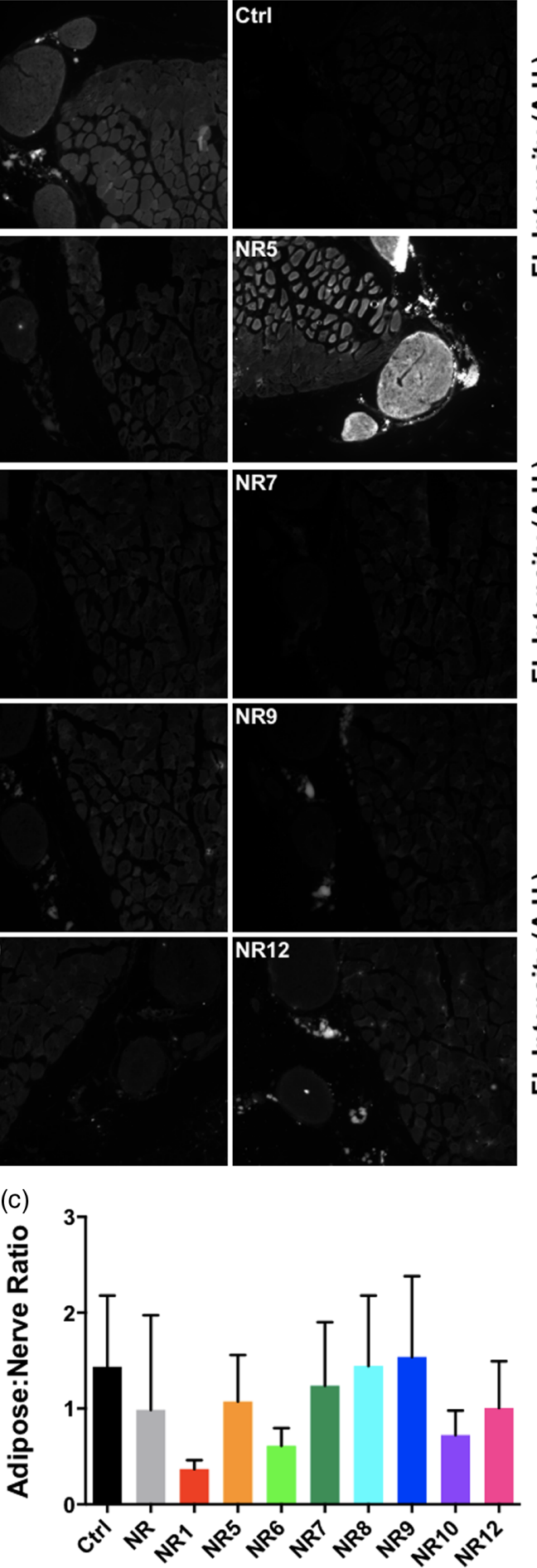

(b)
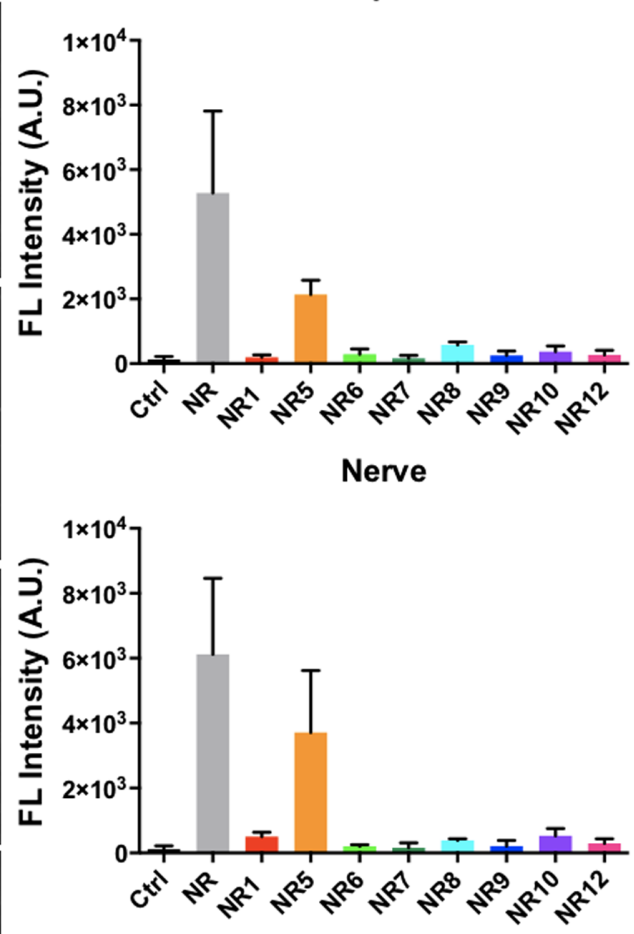

Muscle
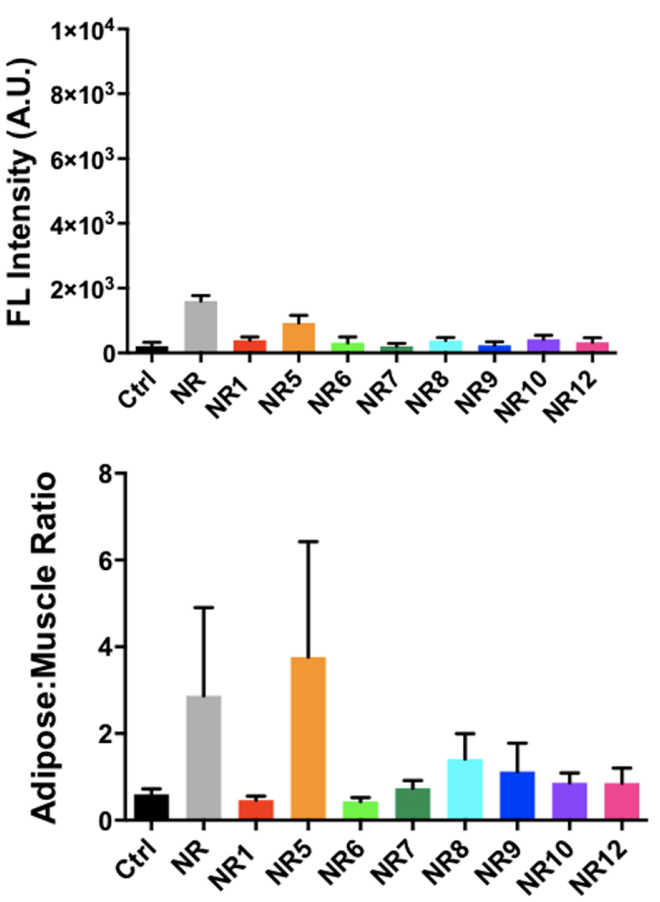

Fig. 6 Ex vivo Nile Red and derivative staining patterns. (a) Nile Red and its synthesized derivatives were used to stain murine tissue sections containing nerve, muscle, and adipose tissues. Images were collected at $650 \mathrm{~ms}$ and representative images are displayed with equivalent normalization. (b) The fluorescence intensity of each tissue type was quantified in $n=4$ tissue sections per fluorophore, where values were used to compute (c) the adipose-to-nerve and adipose-to-muscle ratios. 
washing [Fig. 5(c)]. Fluorescence intensity was generally brightest in this staining study compared to staining of fixed or live cells that were fixed prior to imaging, as determined by the short exposure times. This increase in fluorescence signal was likely due to the lack of washing in the staining procedure. Nile Red was again the brightest overall, with NR5 and NR8 being the brightest of the synthesized derivatives. Prominent lipid droplet-like structures were observed for Nile Red, NR1, NR5, NR9, and NR12. Despite the low quantum yield of NR1, it was again brighter than other Nile Red derivatives with higher quantum yields such as NR12 and NR6 as determined based on optimal exposure time used for image collection.

\subsection{Nile Red Derivative Ex Vivo Tissue Staining}

A subclass of oxazine derivatives with structural similarity to Nile Red and the synthesized derivatives have demonstrated significant nerve specificity. ${ }^{26}$ To determine if any of the structural changes to the Nile Red scaffold in the synthesized derivatives affected the staining specificity for adipose, muscle, or nerve, the synthesized derivatives were used to stain mouse tissue sections containing mouse sciatic nerve as well as the surrounding muscle and adipose tissues (Fig. 6). Nile Red showed adipose specificity as did NR5 and NR12, while little fluorescence staining was seen from the other Nile Red derivatives on mouse tissue sections [Fig. 6(a)]. Region of interest fluorescence intensity analysis showed strong nerve and adipose fluorescence staining from Nile Red and NR5, with substantially lower muscle fluorescence staining intensity [Fig. 6(b)]. The adipose-to-nerve ratio for Nile Red and all of its derivatives was similar to autofluorescence adipose-to-nerve ratio, showing minimal contrast increase from fluorophore staining. Notably, adipose-to-muscle ratio showed increased contrast over control autofluorescence adipose-to-muscle ratio for Nile Red and NR5 [Fig. 6(c)].

\subsection{Nile Red Derivative In Vivo Tissue Staining and Ratiometric Imaging}

To further assess the tissue specificity of the synthesized Nile Red derivatives, in vivo studies were completed where formulated Nile Red and its derivatives were applied to the sciatic and brachial plexus nerves using an optimized direct administration (a)

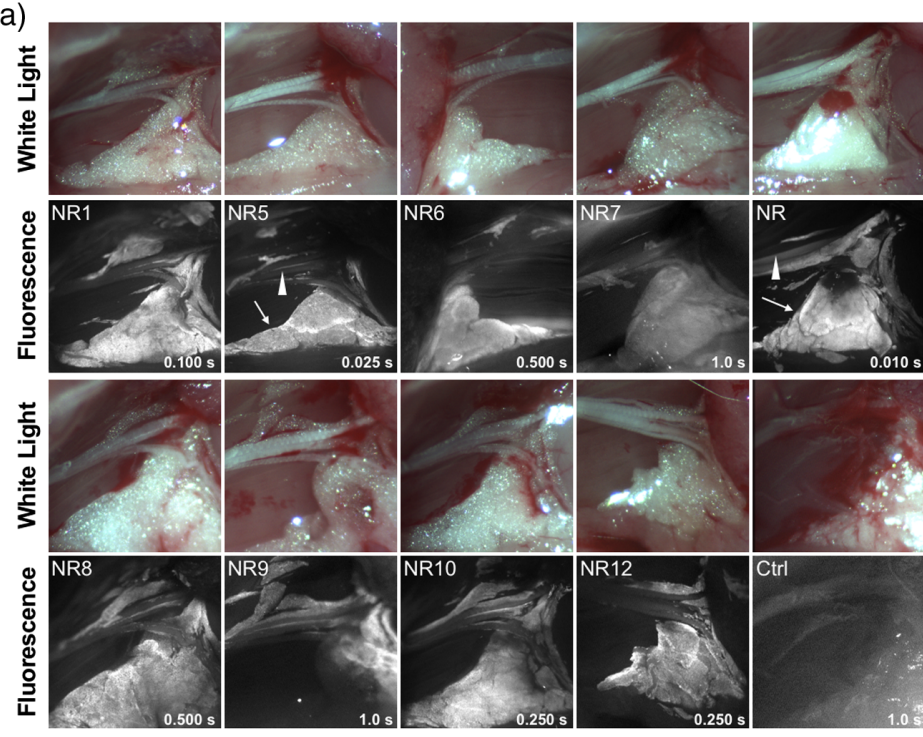

(c)
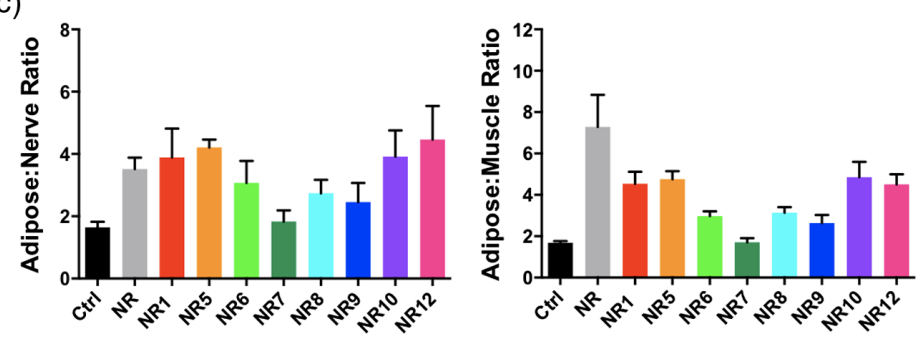
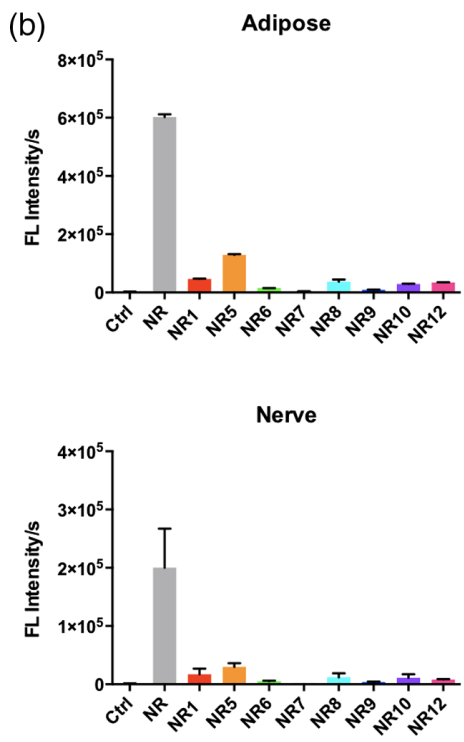

Muscle

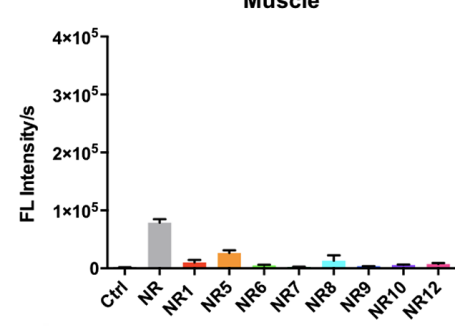

Fig. 7 In vivo tissue-specific Nile Red and derivative staining immediately after direct administration. (a) Nile Red and its synthesized derivatives were used for in vivo direct administration on the brachial plexus and sciatic nerves of mice for $n=4$ nerve sites/fluorophore. Representative images of the sciatic nerve shown in the white light illuminated surgical field of view and their corresponding fluorescence images are shown, where images were collected immediately after completion of staining at the ideal exposure time per fluorophore. Each image is displayed at its ideal contrast. The adipose tissue for NR5 and Nile Red is depicted by the arrow, while the nerve tissue is depicted by the arrowhead. (b) The fluorescence intensities of adipose, muscle, and nerve tissue were quantified for each of the $n=4$ nerve sites/fluorophore, where values were used to compute (c) the adipose-to-nerve and adipose-to-muscle ratios. 
protocol, ${ }^{2}$ which facilitated similar application to the ex vivo frozen tissue section staining. Fluorescence imaging was completed immediately following staining (Fig. 7) and $30 \mathrm{~min}$ after completion of staining (Fig. 8) to determine the utility for contrast during a surgical procedure. In vivo imaging immediately following direct administration showed Nile Red and its derivatives localized to the adipose tissue [Fig. 7(a)]. Tissue-specific fluorescence analysis showed the greatest fluorescence intensity in the adipose tissue, followed by the nerve then muscle tissues. Nile Red and NR5 had the highest fluorescence signal compared to the other Nile Red derivatives, with Nile Red substantially higher than NR5 [Fig. 7(b)]. Adiposeto-nerve ratios showed increased contrast over control autofluorescence with similar adipose-to-nerve contrast from Nile Red, NR1, NR5, NR6, NR10, and NR12. Adipose-to-muscle ratios also showed greater contrast compared to control autofluorescence with the greatest adipose to muscle contrast seen in the Nile Red-stained tissues. While lower than Nile Red, adipose-to-muscle contrast was similar for NR1, NR5, NR10, and NR12 [Fig. 7(c)].

Fluorescence imaging $30 \mathrm{~min}$ after direct administration showed qualitatively similar nerve, adipose, and muscle staining patterns to those observed immediately following direct administration [Figs. 7(a) and 8(a)]. Tissue-specific fluorescence analysis showed a decrease in adipose, nerve, and muscle fluorescence for Nile Red compared to imaging immediately following direct administration. However, NR5 showed slightly increased adipose-specific fluorescence intensity, while nerve and muscle tissue fluorescence were unchanged [Figs. 7(b) and 8(b)]. Adipose-to-nerve contrast was higher than control autofluorescence for Nile Red and all its derivatives except NR7 30 min postdirect administration. Notably, NR5 had higher adipose-to-nerve contrast compared to Nile Red 30 min postdirect administration, while adipose-to-muscle contrast was greatest for Nile Red [Fig. 8(c)]. NR5 tissue-specific contrast was also assessed out to $120 \mathrm{~min}$ after direct administration, where adipose signal was maintained throughout the 120-min imaging time.

To further assess the in vivo adipose- and nerve-specificity differences between Nile Red and NR5, ratiometric imaging was completed using Nile Red or NR5 and oxazine 4 costaining compared to oxazine 4 staining alone [Fig. 9(a)]. ROC curve analysis was completed to quantitatively assess nerve contrast differences between Nile Red and NR5 with oxazine 4 costaining versus oxazine 4 staining alone. AUC calculations from the ROC curve analysis showed similar nerve-to-adipose contrast (a)
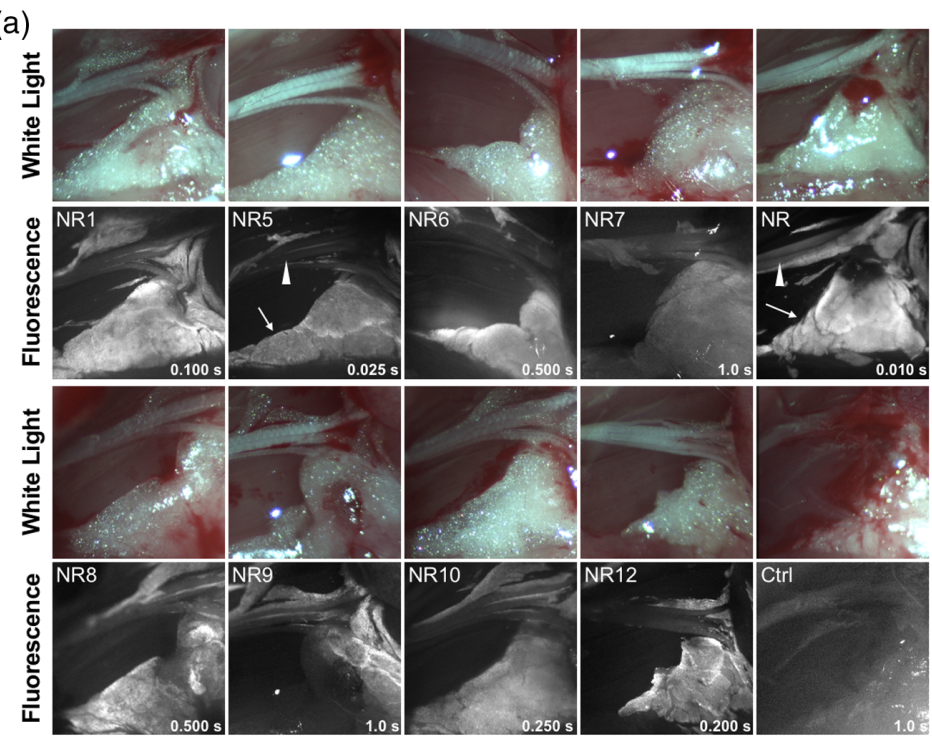

(b)
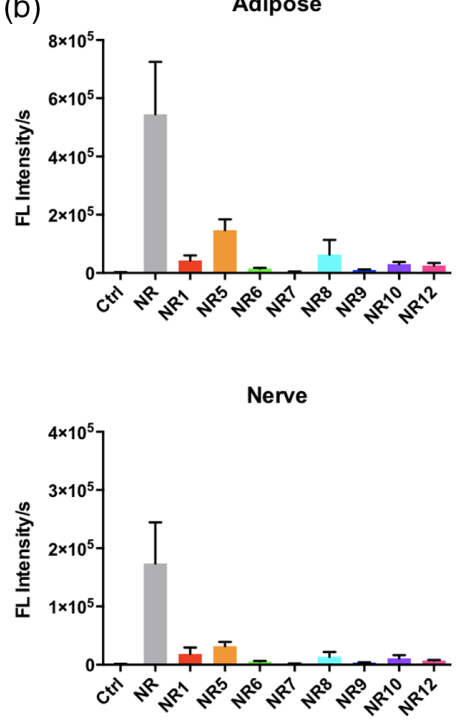

(c)
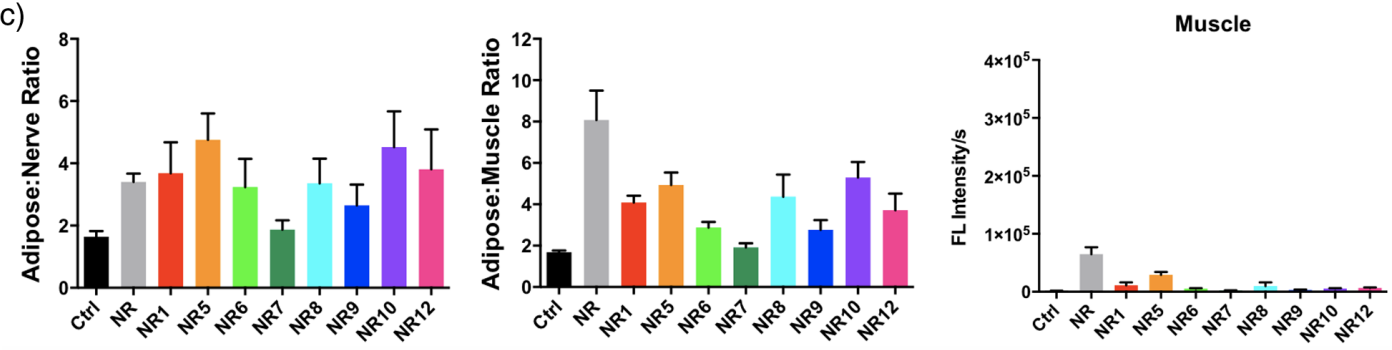

Fig. 8 In vivo tissue-specific Nile Red and derivative staining $30 \mathrm{~min}$ after direct administration. (a) Nile Red and its synthesized derivatives were used for in vivo direct administration on the brachial plexus and sciatic nerves of mice for $n=4$ nerve sites/fluorophore. Representative images of the sciatic nerve shown in the white light illuminated surgical field of view and their corresponding fluorescence images are shown, where images were collected $30 \mathrm{~min}$ after completion of staining at the ideal exposure time per fluorophore. Each image is displayed at its ideal contrast. The adipose tissue for NR5 and Nile Red is shown by the arrow, while the nerve tissue is depicted by the arrowhead. (b) The fluorescence intensities of adipose, muscle, and nerve tissue were quantified for each of the $n=4$ nerve sites/fluorophore, where values were used to compute (c) the adipose-to-nerve and adipose-to-muscle ratios. 


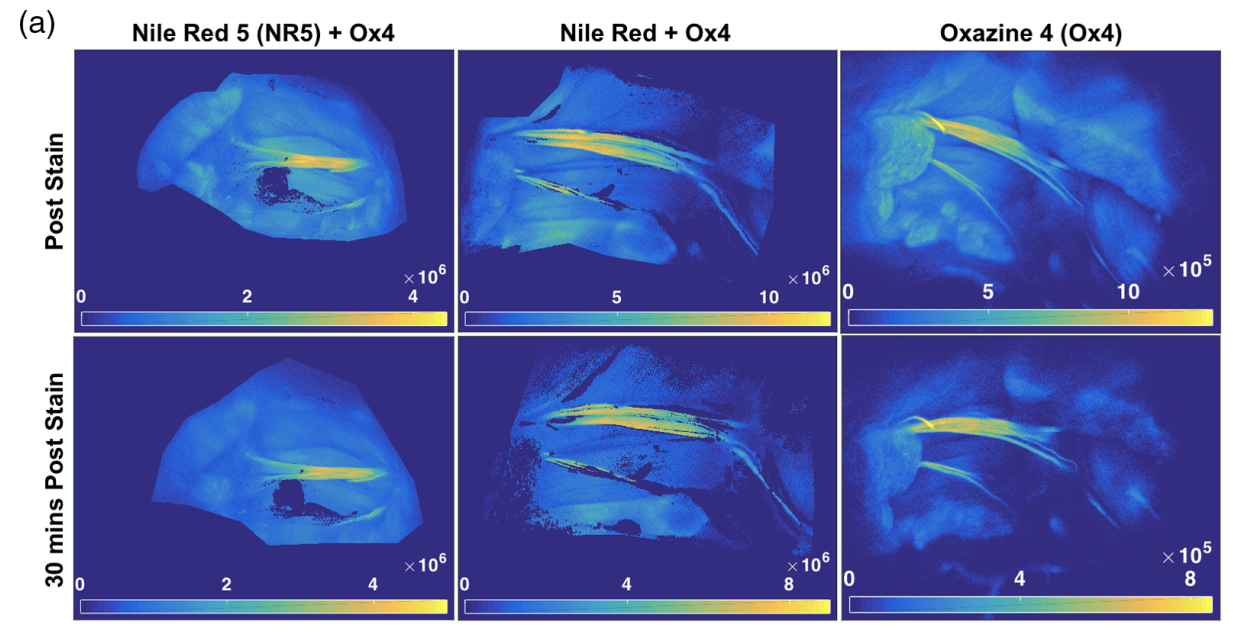

(b)

ROC Area Under the Curve (AUC)

\begin{tabular}{llcc} 
Experiment & & Post Stain & 30 mins Post Stain \\
\hline \multirow{2}{*}{ Nerve:Adipose } & Ox4 & 0.9907 & 0.9966 \\
& NR+Ox4 & 0.9940 & 0.9342 \\
& NR5+Ox4 & 0.9998 & 0.9998 \\
& & & \\
& & & \\
Nerve:Muscle & NR+Ox4 & 0.9744 & 0.9934 \\
& NR5+Ox4 & 0.9712 & 0.8755 \\
& & 0.9845 & 0.9807 \\
\hline
\end{tabular}

Fig. 9 In vivo ratiometric tissue-specific staining after direct administration. (a) Ratiometric images were calculated following in vivo costaining with (left) NR5 and oxazine 4 or (middle) Nile Red and oxazine 4. For contrast comparison, staining was also completed with (right) oxazine 4 alone. (b) ROC curve analysis was performed to assess differences between the ratiometric images and the oxazine 4 alone staining, where AUC was used as the metric to differentiate between the varied staining conditions.

ratios for costaining using oxazine 4 with NR5 or Nile Red or oxazine 4 staining alone immediately following direct administration [Fig. 9(b)]. Although all AUC values were $>0.95$, the differences in nerve-to-adipose contrast using ratiometric imaging compared to oxazine 4 staining alone were found to be statistically significant $(z$-score $=25.26$ for NR versus NR5 costaining with oxazine $4, z$-score $=28.63$ for oxazine 4 only staining versus NR5 costaining with oxazine 4). ROC AUC analysis demonstrated that NR5 and oxazine 4 costaining provided significantly higher nerve-to-adipose contrast than Nile Red and oxazine 4 costaining or oxazine 4 staining alone $30 \mathrm{~min}$ after staining was completed $(z$-score $=98.20$ for $\mathrm{NR}$ versus NR5 costaining with oxazine $4, z$-score $=16.12$ for oxazine 4 only staining versus NR5 costaining with oxazine 4 ).

ROC AUC analysis of nerve-to-muscle contrast immediately following staining showed slightly improved values for NR5 costaining with oxazine 4 compared to Nile Red and oxazine 4 costaining or oxazine 4 only staining. Calculation of the $z$ score revealed that the difference in AUC for NR5 and oxazine 4 costaining verus either Nile Red and oxazine 4 costaining or oxazine 4 alone were statistically significant $(z$-score $=21.87$ for NR versus NR5 costaining with oxazine 4, $z$-score $=$ 15.22 for oxazine 4 only staining versus NR5 costaining with oxazine 4). ROC AUC analysis of the nerve-to-muscle contrast $30 \mathrm{~min}$ after staining revealed little change for NR5 and oxazine 4 costaining and improved values for oxazine 4 staining alone, while Nile Red and oxazine 4 costaining show a substantial drop in AUC value. Calculation of the $z$-score showed that NR5 costaining with oxazine 4 had significantly better nerve-tomuscle contrast compared to Nile Red costaining with oxazine 4 $(z$-score $=99.96)$. However, nerve-to-muscle contrast was not significantly better $30 \mathrm{~min}$ after staining with NR5 and oxazine 4 costaining versus oxazine 4 alone $(z$-score $=-21.32)$.

\section{Discussion}

Current surgical techniques rely heavily on a surgeons' ability to identify normal and diseased tissues using visual and tactile cues during surgery, where outcome is strongly influenced by a surgeon's experience and skill level. The peripheral nervous system is responsible for voluntary and involuntary activities and critically important to bodily physical functions and maintenance of biochemical homeostasis. ${ }^{35}$ Thus, surgical peripheral nerve damage can be devastating with potentially lifelong, deleterious ramifications for affected patients' quality of life. Fluorescenceguided surgery provides an opportunity for rapid, high contrast identification of specific tissue types, such as nerves. However, there are currently few clinically approved fluorophores, none of which are nerve specific. ${ }^{7,13}$ The oxazine fluorophore scaffold presents a unique chemical scaffold for tissue-specific 
fluorescence as modifications to its backbone structure result in both nerve- and adipose-specific fluorescent contrast. ${ }^{18,22,23}$ Accurate intraoperative nerve identification using nerve-specific fluorophores is often hampered by surrounding adipose tissue, since the molecular composition of nerve and adipose tissues is similar. ${ }^{2,13}$ Therefore, small molecule fluorophores that distribute and accumulate in nerve tissue often demonstrate some degree of off target adipose accumulation and contrast. This is particularly problematic for some classes of nerve-specific small molecules including the stilbene derivatives, ${ }^{10}$ distyrylbenzene derivatives, ${ }^{12-15}$ and FM fluorophores. ${ }^{16,17}$ To improve nerve-specific fluorescent contrast for intraoperative guidance, a library of oxazine-based, adipose-specific fluorophores was designed and synthesized to enable ratiometric imaging for enhancement of nerve-specific intraoperative contrast when used as a costain with nerve-specific fluorophores that demonstrate mixed nerve and adipose accumulation.

The Nile Red scaffold was chosen as the backbone fluorophore for library synthesis due to its known lipophilicity- and adipose-highlighting properties. While Nile Red itself does provide adipose-specific fluorescence, it also stains nerve tissue, diminishing nerve-to-adipose tissue contrast by ratiometric imaging [Figs. 7(a)-9(a)]. The Nile Red scaffold was modified (Figs. 1-3) to diminish nerve specificity while maintaining the adipose specificity for improved nerve contrast using a ratiometric imaging approach. While agents with decreased nerve accumulation were generated, their excitation and emission fell outside the ideal NIR range for in vivo imaging (Fig. 4). Similarly, oxazine 4, which was used here as the nerve-specific fluorophore, also has excitation and emission outside of the NIR range as well as minimal adipose accumulation. Thus, in the current studies these agents were used for proof of concept to demonstrate that ratiometric imaging following costaining with nerve- and adipose-specific fluorophores could improve nerve-specific contrast. However, to enable clinical utility, additional synthesis to generate NIR nerve- and adipose-specific small molecules would be beneficial. Of note, synthesis of NIR nerve-specific fluorophores may result in more mixed nerve and adipose accumulation, highlighting the utility of adipose-specific small molecule fluorophores.

The Nile Red derivatives were screened for tissue specificity ex vivo on rodent sciatic nerve sections to assess the utility of ratiometric imaging for enhanced nerve-specific fluorescent contrast. NR5 demonstrated the brightest overall tissue staining compared to the other Nile Red derivatives; however, Nile Red demonstrated the highest overall tissue fluorescence [Figs. 6(a) and 6(b)]. The utility of the Nile Red derivatives for adiposespecific signal was further assessed following direct administration in vivo immediately after as well as 30 min following the completion of staining [Figs. 7(a) and 8(a)]. In vivo direct administration in rodent sciatic and brachial plexus nerves showed strong fluorescence intensity, following Nile Red staining in the adipose, nerve, and muscle tissues immediately following staining [Fig. 7(b)]. Nile Red staining per tissue decreased over the 30-min period after staining, while NR5 showed increased adipose intensity with unchanged nerve and muscle tissue fluorescence 30 min postdirect administration [Fig. 8(b)]. NR5 showed the highest fluorescence intensity in the adipose, nerve, and muscle tissues of the synthesized Nile Red derivatives both immediately poststain as well as at $30 \mathrm{~min}$ after staining [Figs. 7(b) and 8(b)]. The adipose-to-nerve and adipose-tomuscle ratios were calculated to further assess tissue-specific contrast. The mean adipose-to-nerve ratios were similar for NR1, NR5, NR10, NR12, and the parent Nile Red compound immediately after staining [Fig. 7(c)]. Adipose-to-nerve ratio decreased 30 min after staining for Nile Red, while it increased for NR5 demonstrating the potential utility of this agent to highlight adipose-tissue specificity in the context of nerves [Figs. 7(c) and 8(c)]. The change in the adipose-to-nerve ratio was largely due to an overall decrease in nerve and adipose fluorescence signal in the Nile Red group and an increase in the adipose signal for the NR5 group improving overall contrast [Figs. 7(b) and 8(b)]. Adipose-to-muscle signal was greatest for Nile Red compared to all Nile Red derivatives immediately poststain as well as up to $30 \mathrm{~min}$ following staining [Figs. 7(c) and $8(\mathrm{c})$ ].

Direct administration using Nile Red and oxazine 4 costaining was previously optimized, demonstrating adipose- and nerve-specific staining, respectively; however, Nile Red accumulation in the nerve tissue was visible hindering ratiometric analysis for improved nerve contrast. ${ }^{2}$ NR5 was selected for ratiometric imaging studies due to its tissue-specific staining and adipose-to-nerve contrast to determine if contrast was improved over Nile Red and oxazine 4 costaining or oxazine 4 staining alone. Ratiometric images using both Nile Red and NR5 to specifically highlight the adipose tissue and oxazine 4 to highlight nerve tissue resulted in high contrast immediately poststain as well as $30 \mathrm{~min}$ after staining [Fig. 9(a)]. ROC curve analysis showed the most significant improvement in nerve-toadipose contrast $30 \mathrm{~min}$ poststaining, where the AUC was decreased for Nile Red and oxazine 4 costaining but maintained for NR5 and oxazine 4 costaining [Fig. 9(b)]. Notably, the nerve-to-muscle contrast did not show the same level of improvement as nerve-to-adipose contrast using either Nile Red or NR5 costaining with oxazine 4 , which was likely due to the fact that oxazine 4 does not show substantial adipose or muscle accumulation. ROC curve analysis showed similar AUC immediately poststaining, where a statistically significant improvement was seen using NR5 costaining with oxazine 4 versus oxazine 4 alone. However, at 30 min poststaining, the nerve-to-muscle contrast was not statistically different between NR5 and oxazine 4 costaining versus oxazine 4 staining alone. This points to a limitation of the current compounds, where only nerve-to-adipose contrast would be improved during the surgical time frame using the dual staining technique whereas nerveto-muscle contrast would not benefit. Additional synthetic optimization is ongoing to generate an adipose-specific derivative that can both reach NIR wavelengths and improve nerve-tomuscle contrast, justifying the use of two small molecule fluorophores instead of a single agent for nerve-specific contrast clinically.

In summary, a Nile Red derivative, NR5, was synthesized and characterized for its adipose specificity as a proof of concept small molecule to enable ratiometric imaging for improved nerve-specific contrast. NR5 showed both adipose specific contrast as well as minimal nerve accumulation, differentiating it from the parent compound Nile Red, which demonstrates some nerve accumulation. In vivo costaining with nerve-specific oxazine 4 and NR5 showed improved nerve-to-adipose contrast as compared to the parent Nile Red compound due to this decreased nerve accumulation. This contrast improvement was most apparent 30 min postdirect administration, demonstrating the potential utility for surgical applications. These proof of concept studies show the potential utility of ratiometric 
imaging to improve contrast in tissues such as nerve, where development of a tissue-specific contrast agent that does not accumulate in other surrounding tissue is a continued challenge.

\section{Disclosure}

The authors declare no competing financial interests.

\section{Acknowledgments}

We would like to thank Sarah Cook and Evan Muschler for experimental assistance. This work was funded by the National Cancer Institute (R01CA188491) and the National Institute for Biomedical Imaging and Bioengineering (R01EB021362).

\section{References}

1. W. A. Macrae, "Chronic post-surgical pain: 10 years on," Br. J. Anaesth. 101, 77-86 (2008).

2. C. W. Barth and S. L. Gibbs, "Direct administration of nerve-specific contrast to improve nerve sparing radical prostatectomy," Theranostics 7, 573-593 (2017).

3. S. Keereweer et al., "Optical image-guided surgery: where do we stand?" Mol. Imaging Biol. 13, 199-207 (2011).

4. A. V. DSouza et al., "Review of fluorescence guided surgery systems: identification of key performance capabilities beyond indocyanine green imaging," J. Biomed. Opt. 21, 080901 (2016).

5. F. Acerbi et al., "Fluorescein-guided surgery for malignant gliomas: a review," Neurosurg. Rev. 37, 547-557 (2014).

6. M. S. Eljamel and S. O. Mahboob, "The effectiveness and cost-effectiveness of intraoperative imaging in high-grade glioma resection; a comparative review of intraoperative ALA, fluorescein, ultrasound and MRI," Photodiagn. Photodyn. Ther. 16, 35-43 (2016).

7. S. L. Gibbs, "Near infrared fluorescence for image-guided surgery," Quant. Imaging Med. Surg. 2, 177-187 (2012).

8. S. Gioux, H. S. Choi, and J. V. Frangioni, "Image-guided surgery using invisible near-infrared light: fundamentals of clinical translation," Mol. Imaging 9, 237-255 (2010).

9. A. L. Vahrmeijer et al., "Image-guided cancer surgery using near-infrared fluorescence," Nat. Rev. Clin. Oncol. 10, 507-518 (2013).

10. C. Wu et al., "Molecular probes for imaging myelinated white matter in CNS," J. Med. Chem. 51, 6682-6688 (2008).

11. C. Wang et al., "In situ fluorescence imaging of myelination," J. Histochem. Cytochem. 58, 611-621 (2010).

12. V. E. Cotero et al., "Intraoperative fluorescence imaging of peripheral and central nerves through a myelin-selective contrast agent," Mol. Imaging Biol. 14, 708-717 (2012).

13. S. L. Gibbs et al., "Structure-activity relationship of nerve-highlighting fluorophores," PLoS One 8, e73493 (2013).

14. S. L. Gibbs-Strauss et al., "Nerve-highlighting fluorescent contrast agents for image-guided surgery," Mol. Imaging 10, 91-101 (2011).

15. B. Stankoff et al., "Imaging of CNS myelin by positron-emission tomography," Proc. Natl. Acad. Sci. U. S. A. 103, 9304-9309 (2006).

16. J. R. Meyers et al., "Lighting up the senses: FM1-43 loading of sensory cells through nonselective ion channels," J. Neurosci. 23, 4054-4065 (2003).
17. S. L. Gibbs-Strauss et al., "Molecular imaging agents specific for the annulus fibrosus of the intervertebral disk," Mol. Imaging 9, 128140 (2010).

18. M. H. Park et al., "Prototype nerve-specific near-infrared fluorophores," Theranostics 4, 823-833 (2014).

19. C. Wang et al., "Longitudinal near-infrared imaging of myelination," J. Neurosci. 31, 2382-2390 (2011).

20. U. Fagerholm, "The highly permeable blood-brain barrier: an evaluation of current opinions about brain uptake capacity," Drug Discov. Today 12(23-24), 1076-1082 (2007).

21. R. N. Waterhouse, "Determination of lipophilicity and its use as a predictor of blood-brain barrier penetration of molecular imaging agents," Mol. Imaging Biol. 5, 376-389 (2003).

22. P. Greenspan, E. P. Mayer, and S. D. Fowler, "Nile red: a selective fluorescent stain for intracellular lipid droplets," J. Cell Biol. 100, 965-973 (1985).

23. V. Martinez and M. Henary, "Nile red and nile blue: applications and syntheses of structural analogues," Chemistry 22, 13764-13782 (2016).

24. R. Wei et al., "Nile red derivative-modified nanostructure for upconversion luminescence sensing and intracellular detection of $\mathrm{Fe}(3+)$ and $\mathrm{MR}$ imaging," ACS Appl. Mater. Interfaces 8, 400-410 (2016).

25. R. A. Velapoldi and H. H. Tonnesen, "Corrected emission spectra and quantum yields for a series of fluorescent compounds in the visible spectral region," J. Fluoresc. 14, 465-472 (2004).

26. W. Connor and S. L. G. Barth, "Visualizing oxazine 4 nerve-specific fluorescence ex vivo in frozen tissue sections," Proc. SPIE 9696, 96960R (2016).

27. K. M. Hackman et al., "Polymeric micelles as carriers for nerve-highlighting fluorescent probe delivery," Mol. Pharm. 12(12), 4386-4394 (2015)

28. C. W. Barth et al., "Optimizing fresh specimen staining for rapid identification of tumor biomarkers during surgery," Theranostics 7, 47224734 (2017)

29. J. A. Hanley and B. J. McNeil, "A method of comparing the areas under receiver operating characteristic curves derived from the same cases," Radiology 148, 839-843 (1983).

30. M. S. J. Briggs et al., "Synthesis of functionalised fluorescent dyes and their coupling to amines and amino acids," J. Chem. Soc. Perkin Trans. 1 1051-1058 (1997).

31. M. J. Mio et al., "One-pot synthesis of symmetrical and unsymmetrical bisarylethynes by a modification of the sonogashira coupling reaction," Org. Lett. 4, 3199-3202 (2002).

32. N. Miyaura and A. Suzuki, "Palladium-catalyzed cross-coupling reactions of organoboron compounds," Chem. Rev. 95, 2457-2483 (1995).

33. A. F. Littke, C. Dai, and G. C. Fu, "Versatile catalysts for the suzuki cross-coupling of arylboronic acids with aryl and vinyl halides and triflates under mild conditions," J. Am. Chem. Soc. 122, 4020-4028 (2000).

34. C. Reichardt, "Solvatochromic dyes as solvent polarity indicators," Chem. Rev. 94, 2319-2358 (1994).

35. G. P. Chrousos and P. W. Gold, "The concepts of stress and stress system disorders. overview of physical and behavioral homeostasis," J. Am. Med. Assoc. 267, 1244-1252 (1992).

Biographies for the authors are not available. 